\title{
Up-regulated Expression of MTHFD2 Correlates with Favorable Prognosis in LGG Patients: A Bioinformatic Analysis
}

\section{Lu-feng Shi}

Zhejiang University School of Medicine Sir Run Run Shaw Hospital

Qian Zhang

Zhejiang University School of Medicine Sir Run Run Shaw Hospital

\section{Xiao-ying Shou}

Zhejiang University School of Medicine Sir Run Run Shaw Hospital

Huan-jiang Niu (D 3203022@zju.edu.cn )

Zhejiang University School of Medicine Sir Run Run Shaw Hospital

\section{Research}

Keywords: MTHFD2, lower-grade glioma, high expression, prognosis

Posted Date: October 26th, 2020

DOl: https://doi.org/10.21203/rs.3.rs-96103/v1

License: (a) (i) This work is licensed under a Creative Commons Attribution 4.0 International License.

Read Full License 


\title{
Up-regulated expression of MTHFD2 correlates with favorable prognosis in LGG patients: a bioinformatic analysis
}

\author{
Lu-feng Shi ${ }^{1}$, Qian Zhang ${ }^{1}$, Xiao-ying Shou ${ }^{2}$, Huan-jiang Niu ${ }^{1 *}$ \\ ${ }^{1}$ Department of Neurosurgery, Sir Run Run Shaw Hospital, Zhejiang University \\ School of Medicine, Hangzhou 310016, Zhejiang, China \\ ${ }^{2}$ Department of Nursing, Sir Run Run Shaw Hospital, Zhejiang University School of \\ Medicine, Hangzhou 310016, Zhejiang, China
}

*Corresponding author: Huan-jiang Niu

Address: Department of Neurosurgery, Sir Run Run Shaw Hospital, Zhejiang University School of Medicine, 368 Xiasha Road, Hangzhou, Zhejiang, 310016, China.

Email: 3203022@zju.edu.cn 
Abstract
Background Methylenetetrahydrofolate dehydrogenase 2 (MTHFD2) was found to be up-regulated in various cancers and has been identified as a potential target for cancer treatment, but the potential function of MTHFD2 in low-grade gliomas (LGG) has not been well-examined. This study initially revealed the potential function of MTHD2 and emphasized its importance in LGG.

Methods We first explored the expression of MTHFD2 in LGG patients using Oncomine and UALCAN database. Survival analysis on LGG patients was then conducted based on age, gender, radiation therapy, histologic grade, tumor type and MTHFD2 expression. Cox regression analysis was also applied to predict the prognostic factor for overall survival (OS) of LGG. Finally, we performed enrichment analysis to reveal the potential MTHFD2-associated pathways involved in LGG.

Results We found that MTHFD2 is highly expressed in LGG patients, and MTHFD2 expression is related with age, sub-types and TP53-mutation status (all $P<0.05$ ). Age, radiation therapy, histologic grade and tumor type were implicated in the survival of LGG patients (all $P<0.05$ ). High expression of MTHFD2 desirably improved the prognosis of LGG patients $(P<0.001)$, especially for those patients with characteristics of age $\geq 45$ years old $(P<0.001)$, receiving radiation therapy treatment $(P=0.001)$, sub-type of astrocytoma and oligodendroglioma (all $P<0.05)$, and histologic grade $3(P<0.05)$. MTHFD2 was determined as an independent prognostic factor with age and grade for OS of LGG patients (all $P<0.01$ ). Pathway enrichment analysis indicated that MTHFD2 mainly participates in mTOR signaling pathway, apelin pathway and folate-mediated one-carbon metabolism.

Conclusions The expression of MTHFD2 is associated with key clinical phenotype. High expression of MTHFD2 is favorable for the overall survival of LGG patients. MTHFD2 may serve as a novel prognostic biomarker and potential therapeutic target for LGG treatment.

Key words: MTHFD2; lower-grade glioma; high expression; prognosis 


\section{Introduction}

Glioma is the most common malignant tumor in the central nervous system, accounting for $70 \%$ of brain cancers [1]. According to the latest statistic reports in China [2], 101,600 new cases and 61,000 related deaths have appeared. The World Health Organization classified gliomas into grade 1 to grade 4 based on histology and clinical criteria [3]. Low-grade gliomas (LGG), belonging to grade 2 tumor, accounts for $15 \%-25 \%$ of all gliomas and includes astrocytomas, oligoastrocytomas and oligodendrogliomas [4]. LGG is relatively benign as well as slow-growing, and overall survival (OS) of children and adolescents with LGG exceeds $90 \%$ at 5 to 10 years [5]. Despite growing slowly initially, essentially all LGG lesions eventually progress to high-grade glioma (HGG) (Grade 3/4) [6, 7]. Approximately 50\% of patients with LGGs will experience malignant transformation within 5 years [8]. Hence, identification of potential therapeutic target or biomarker is desirable to precisely predict the prognosis of LGG patients and improve their clinical outcome.

Methylenetetrahydrofolate dehydrogenase 2 (MTHFD2) is a mitochondrial enzyme involved in folate metabolism [9]. MTHFD2 is also a bifunctional enzyme with methylene dehydrogenase and cyclohydrolase activities [10]. Growing evidences demonstrated the up-regulation of MTHFD2 in various cancers and its over-expression was associated with tumour cell proliferation [11]. Inhibition of MTHFD2 expression affected proliferation of various cancer cells including lung [12], breast [13] and acute myeloid leukemia [14]. These studies also highlighted MTHFD2 as being a promising therapeutic target for cancer treatment. For instance, MTHFD2 was identified as the drug target to block breast cancer cell migration and invasion [15]. Currently, it has been regarded as one of the candidate marker genes for tumor invasion, metastasis and poor prognosis [9]. Despite the growing interest about MTHFD2 as a potential target in cancers, the potential function of this enzyme in LGG has not been defined.

The present study was aimed at initially revealing the potential function of MTHFD2 in LGG. We first explored the MTHFD2 expression in LGG based under different aspects. The influence on prognosis of LGG patients and prognostic value on OS were then evaluated through survival analysis. Finally, MTHFD2-associated pathway analysis was performed to understand the potential mechanism in LGG.

\section{Methods}

\subsection{MTHFD2 expression analysis}

We first used Oncomine database to explore the expression of MTHFD2 in human cancers. The threshold was set as $P$-value: 0.05 , fold change: 2 and gene rank: TOP $10 \%$. The expression of MTHFD2 in brain cancer and normal tissues was also assessed according to previous studies.

The MTHFD2 expression based on age, gender, race, tumor grade, sub-types and TP53 mutation status in LGG patients was further evaluated through UALCAN database. Due to the importance of methylation and TP53 expression in cancers, the influences of MTHFD2 methylation and TP53 expression on MTHFD2 expression were estimated as well.

2.2 Prognosis analysis on LGG patients

We then evaluated the association of clinical phenotype with OS of LGG patients by Kaplan-Meier analysis and log-rank test. Clinical phenotype contained age, gender, radiation therapy, histologic grade and tumor type. Gepia database was applied to analyze the MTHFD2 expression on the prognosis of LGG patients including OS and disease free survival (DFS). The 
LGG patients were divided into high- and low-expression groups according to the median of MTHFD2 expression. Subsequently, the influence of MTHFD2 expression was taken into consideration for OS analysis of LGG patients based on different clinical phenotype. The clinical data of LGG patients and expression profile of MTHFD2 were obtained from cBioportal database.

2.3 Functional enrichment analysis

We finally conducted functional enrichment analyses, including Gene Ontology (GO) and Kyoto Encyclopedia of Genes and Genomes (KEGG) pathway analyses, to identify MTHFD2-related biological functions and signaling pathways. The co-expressed genes with MTHFD2 were found through cBioportal database. Identified genes positively and negatively correlated with MTHFD2, as threshold by absolute correlation $>0.5$ and $P<0.05$, were selected for further GO and KEGG analysis, respectively. GO terms, classifying into molecular function (MF), biological process (BP) and cellular component (CC), were annotated via DAVID database. KEGG pathway analysis was conducted by KEGG Orthology-Based Annotation System (KOBAS).

\subsection{Statistical analysis}

All the statistical analyses were performed using SPSS 19.0. Mann-Whitney U test was performed for comparisons between the two groups. The Kruskal-Wallis test was performed for multiple comparisons followed by Bonferroni's post-hoc test. $P$ value less than 0.05 was regarded as statistical significance.

\section{Results}

\subsection{The over-expression of MTHFD2 in human cancers}

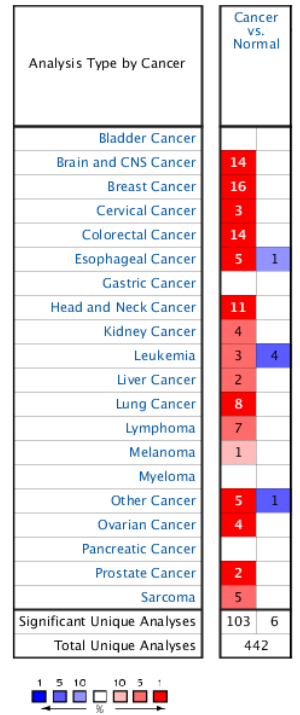

(A)
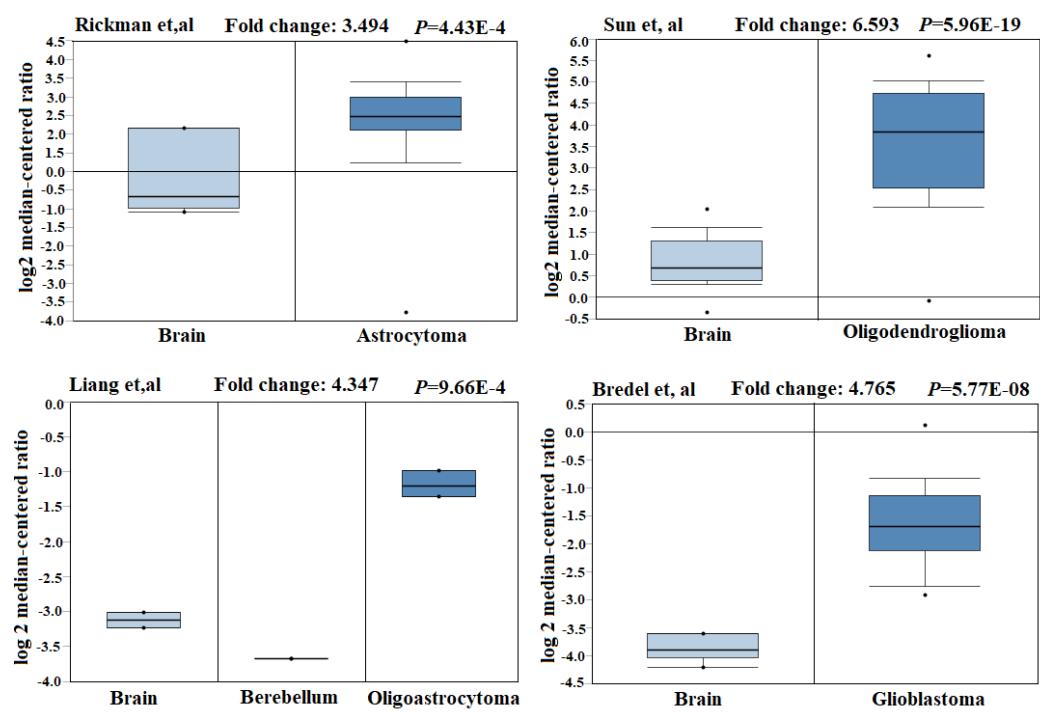

(B)

Fig.1 Expression analysis on MTHFD2. (A) disease summary for MTHFD2; (B) MTHFD2 expression in normal and brain cancers. The threshold was set by $P$-value: 0.05 , fold change: 2 , gene rank: TOP $10 \%$. The data was obtained from Oncomine database.

We first analyzed the mRNA expression of MTHFD2 in various human cancers (Fig.1A). Compared with normal tissues, MTHFD2 was highly expressed in some cancers such as brain and CNS cancer, breast cancer and colorectal cancer. Low expression of MTHFD2 was reported in esophageal cancer and leukemia. 
The expression of MTHFD2 in brain cancer and normal samples was further evaluated based on previous researches (Fig.1B). We found that the expression degree of MTHFD2 differs among sub-types of brain cancers, which indicted the different role of MTHFD2 in different brain cancers.

\subsection{The expression of MTHFD2 in LGG}

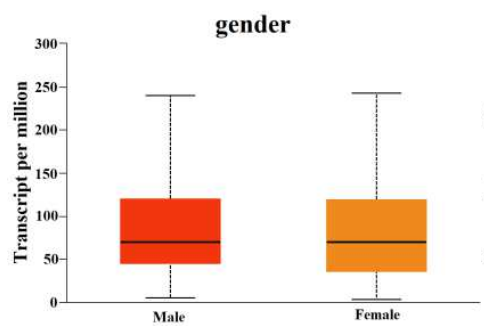

(A)

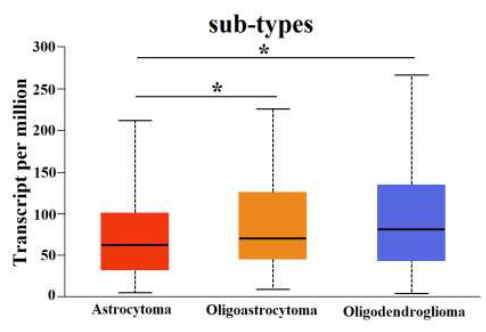

(D)

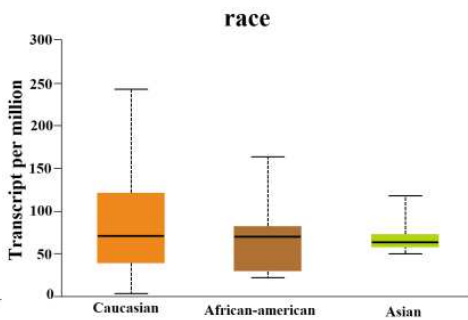

(B)

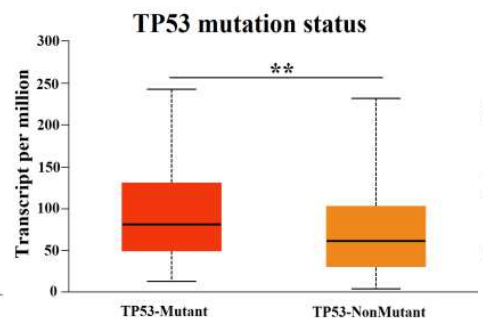

(E)

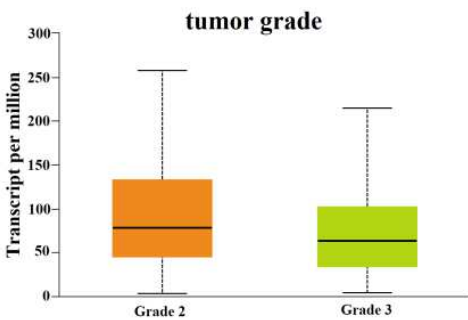

(C)

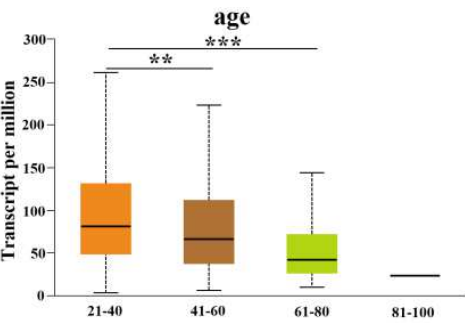

(F)

Fig.2 MTHFD2 expression profile based on clinical phenotype in LGG. (A) gender; (B) race; (C) tumor grade; (D) sub-types; (E) TP53 mutation status; (F) age. ${ }^{*} P<0.05,{ }^{* * *} P<0.01$ and ${ }^{* * *} P<0.001$.

We then explored the association of clinical phenotype in LGG with MTHFD2 expression. The results suggested that the expression of MTHFD2 is not associated with gender, race and tumor grade of LGG patients (Fig.2A-2C). The sub-types of brain cancers influenced the MTHFD2 expression and the lowest expression was observed in astrocytoma (Fig.2D, all $P<0.05$ ). The expression of MTHFD2 was also related with TP53 mutation status, and TP53 mutant led to higher MTHFD2 expression (Fig.2E, $P<0.01$ ). In addition, the MTHFD2 expression statistically differed in different age, and low age showed higher expression of MTHFD2 (Fig.2F, all $P<0.01$ ).

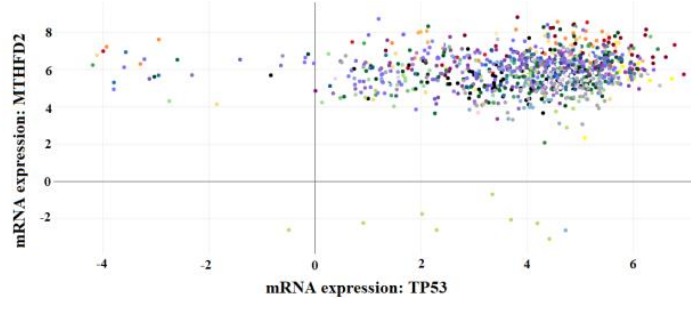

(A)

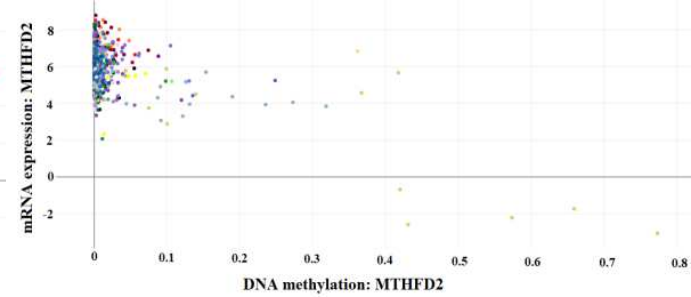

(B)

Fig.3 The expression of MTHFD2 with (A) TP53 expression and (B) MTHFD2 methylation.

The influences of MTHFD2 methylation and TP53 expression on MTHFD2 expression were assessed as well. Fig.3A shows that the expression of MTHFD2 has no obvious change with the increase of TP53 expression. Fig.3B indicates that MTHFD2 is in low-methylation which exerts no effects on its own mRNA expression. 


\subsection{Prognosis analysis in LGG patients}

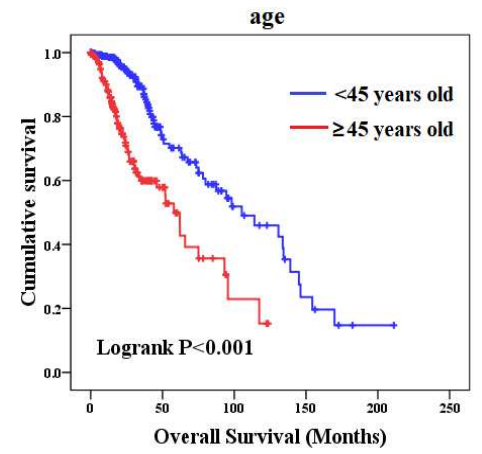

(A)

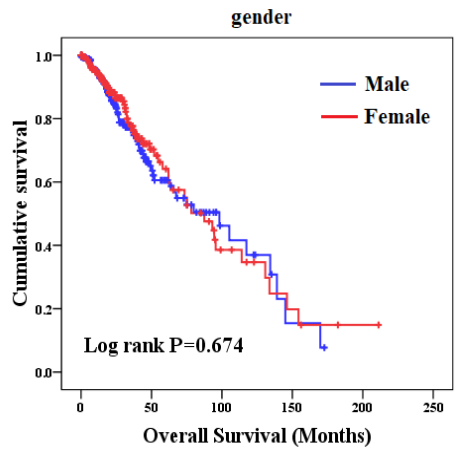

(B)

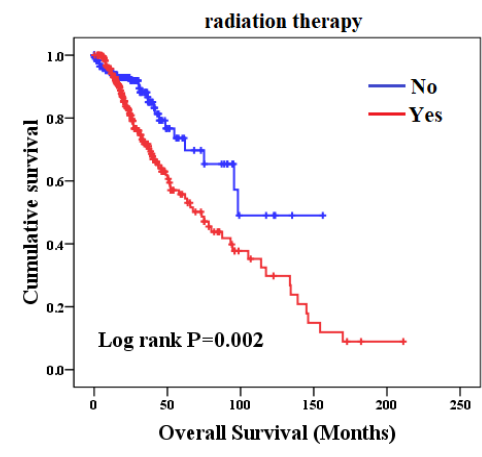

(C)

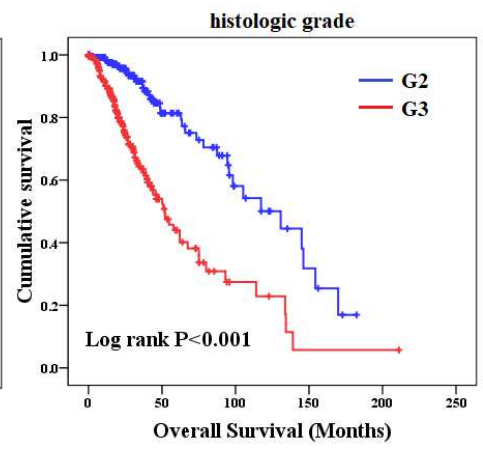

(D)

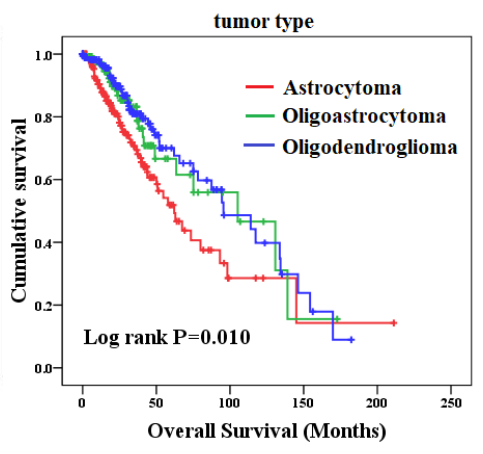

(E)

Fig.4 The association of clinical phenotype with OS in LGG patients. (A) age; (B) gender; (C) radiation therapy; (D) histologic grade; (E) tumor type.

The association of clinical phenotype with overall survival of LGG patients was predicted using Kaplan-Meier analysis and log-rank test. Survival analysis indicted that LGG patients with age $\geq 45$ years old shows worse prognosis (Fig.4A, $P<0.001$ ). The overall survival time of male and female patients has no obvious differences (Fig.4B, $P=0.674$ ). The radiation therapy affected the prognosis of LGG patients, and the survival time of patients receiving radiation therapy decreased compared with these patients without radiation therapy treatment (Fig.4C, $P=0.002$ ). Patients in G3 grade showed shorter survival time than patients in G2 grade (Fig.4D, $P<0.001$ ). The prognosis of LGG patients was statistically correlated with tumor types, and patients with astrocytoma displayed the worst prognosis (Fig.4E, $P=0.010$ ).

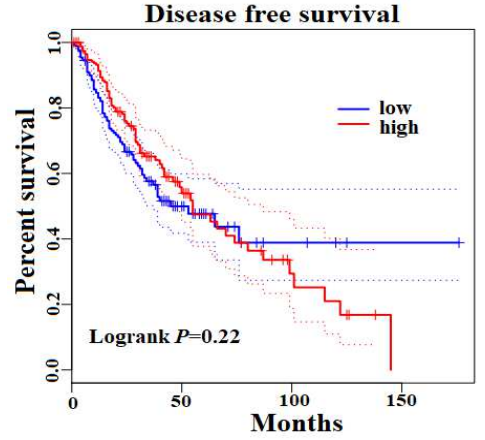

(A)

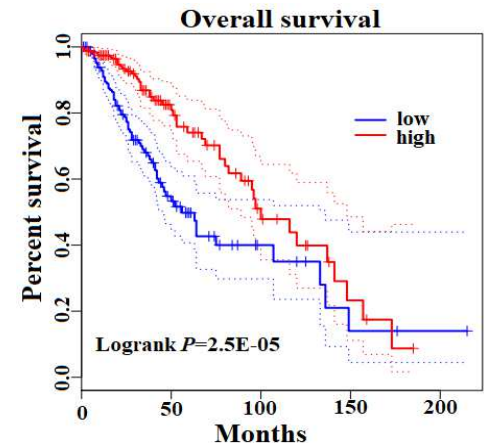

(B)

Fig.5 The association of MTHFD2 expression with prognosis of LGG patients. (A) disease free survival (DFS); (B) Overall survival (OS). 
The effect of MTHFD2 expression on the clinical outcome of LGG patients was predicted as well through Gepia database. Survival analysis suggested that the MTHFD2 expression did not affect the disease free survival of LGG patients (Fig.5A, $P=0.22$ ). However, high expression of MTHFD2 conferred to a favorable prognosis for LGG patients (Fig.5B, $P<0.001$ ), which indicated that MTHFD2 plays a positive role in LGG.
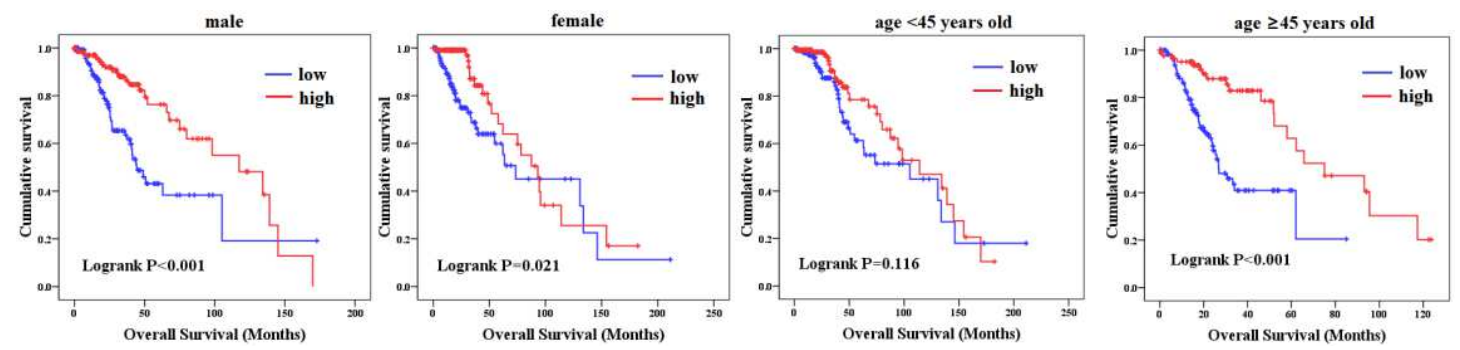

(A)

(B)
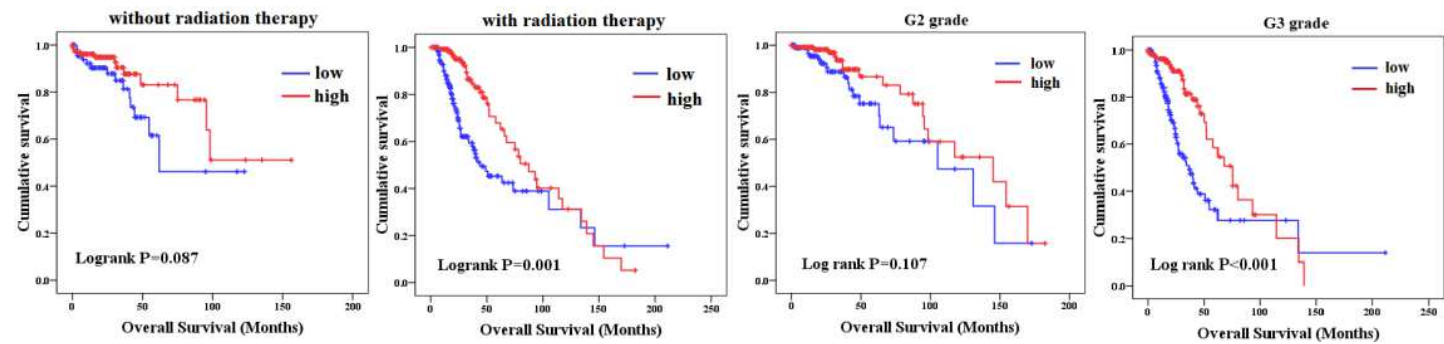

(C)

(D)
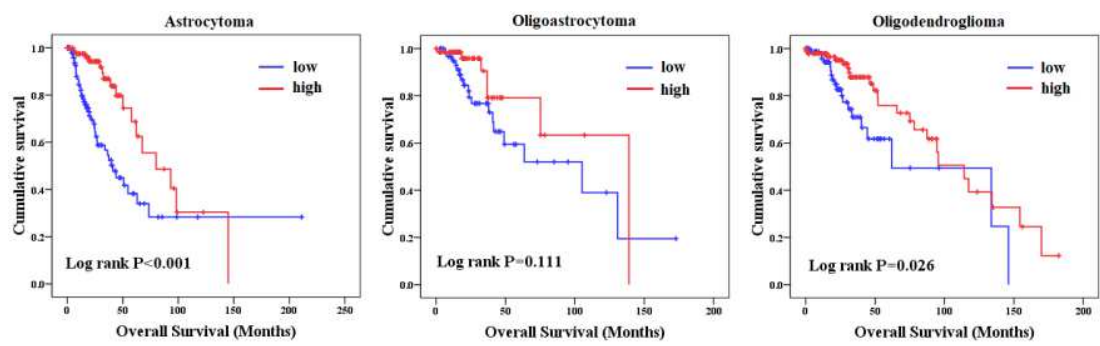

(E)

Fig.6 The association of MTHFD2 expression with OS of LGG patients. (A) gender; (B) age; (C) radiation therapy; (D) histologic grade; (E) tumor type.

In order to reveal the detailed influence of MTHFD2 on the survival of LGG patients, the association of MTHFD2expression with prognosis was assessed under different clinical phenotype. The results indicated that the high expression of MTHFD2 is statistically related with longer survival time in both male and female patients (Fig.6A). MTHFD2 high expression mainly improved the prognosis of patients with age $\geq 45$ years old (Fig.6B). In LGG patients with receiving radiation therapy, MTHFD2 high expression had positive effect on their survival time (Fig.6C). The result also revealed that MTHFD2 low expression mainly shortened the survival time of patients in G3 grade compared with patients in G2 grade (Fig.6D). With regard to sub-types, MTHFD2 expression obviously affected the prognosis of patients with astrocytoma and oligodendroglioma (Fig.6E). 
Table.1 Association of clinical characteristics with OS of LGG patients using Cox regression

\begin{tabular}{ccccccc}
\hline & \multicolumn{3}{c}{ Univariate analysis } & \multicolumn{3}{c}{ Multivariate analysis } \\
& HR & $95 \% C I$ & $P$ & HR & $95 \% C I$ & $P$ \\
\hline age & 2.932 & $2.020-4.256$ & $<0.001$ & 2.924 & $1.970-4.341$ & $<0.001$ \\
MTHFD2 expression & 0.455 & $0.316-0.655$ & $<0.001$ & 0.510 & $0.348-0.747$ & 0.001 \\
histologic grade & 3.324 & $2.247-4.917$ & $<0.001$ & 2.599 & $1.688-4.001$ & $<0.001$ \\
sub-types & 0.747 & $0.609-0.917$ & 0.005 & 0.805 & $0.648-1.001$ & 0.051 \\
radiation therapy & 1.951 & $1.261-3.018$ & 0.003 & 1.117 & $0.695-1.795$ & 0.648 \\
gender & 0.927 & $0.649-1.322$ & 0.674 & 0.842 & $0.569-1.192$ & 0.304 \\
\hline
\end{tabular}

In order to predict the prognostic factor for OS of LGG patients, we performed Cox regression analysis regarding several clinical phenotypes. As suggested in Table.1, age, MTHFD2 expression, histologic grade, sub-types and radiation therapy status were all associated with OS of LGG patients (all $P<0.01$ ) in univariate analysis. Multivariate analysis indicted that MTHFD2 remained as an independent prognostic factor $(\mathrm{HR}=0.510, P=0.001)$ with age $(\mathrm{HR}=2.924$, $P<0.001)$ and histologic grade $(\mathrm{HR}=2.599, P<0.001)$ for OS.

Table.2 Prognosis analysis for OS of LGG patients in different sub-types using multivariate Cox regression

\begin{tabular}{|c|c|c|c|c|c|c|}
\hline & \multicolumn{2}{|c|}{ Astrocytoma } & \multicolumn{2}{|c|}{ Oligoastrocytoma } & \multicolumn{2}{|c|}{ Oligodendroglioma } \\
\hline & $\operatorname{HR}(95 \% C I)$ & $P$ & $\operatorname{HR}(95 \% C I)$ & $P$ & HR (95\%CI) & $P$ \\
\hline age & $\begin{array}{c}3.462 \\
(1.942-6.169)\end{array}$ & $<0.001$ & $\begin{array}{c}2.776 \\
(1.145-6.728)\end{array}$ & 0.024 & $\begin{array}{c}2.636 \\
(1.237-5.618)\end{array}$ & 0.012 \\
\hline $\begin{array}{l}\text { MTHFD2 } \\
\text { expression }\end{array}$ & $\begin{array}{c}0.471 \\
(0.260-0.853)\end{array}$ & 0.013 & $\begin{array}{c}0.407 \\
(0.162-1.025)\end{array}$ & 0.056 & $\begin{array}{c}0.519 \\
(0.256-1.052)\end{array}$ & 0.069 \\
\hline $\begin{array}{l}\text { histologic } \\
\text { grade }\end{array}$ & $\begin{array}{c}4.612 \\
(1.917-11.096)\end{array}$ & 0.001 & $\begin{array}{c}3.034 \\
(1.245-7.396)\end{array}$ & 0.015 & $\begin{array}{c}2.062 \\
(1.019-4.176)\end{array}$ & 0.044 \\
\hline $\begin{array}{l}\text { radiation } \\
\text { therapy }\end{array}$ & $\begin{array}{c}0.559 \\
(0.257-1.212)\end{array}$ & 0.141 & $\begin{array}{c}1.428 \\
(0.478-4.263)\end{array}$ & 0.523 & $\begin{array}{c}1.455 \\
(0.703-3.014)\end{array}$ & 0.313 \\
\hline gender & $\begin{array}{c}0.861 \\
(0.498-1.489)\end{array}$ & 0.592 & $\begin{array}{c}1.439 \\
(0.610-3.396)\end{array}$ & 0.406 & $\begin{array}{c}0.616 \\
(0.315-1.203)\end{array}$ & 0.156 \\
\hline
\end{tabular}

Due to the significant effect of sub-types on the prognosis of LGG patients, we further predicted the prognostic factor for OS of LGG patients under different sub-types. The results indicated that age and histologic grade are the common factors for predicting prognosis of three sub-types (Table.2). However, the expression of MTHFD2 was just an independent prognostic factor for $\mathrm{OS}$ of patients with astrocytoma (HR=0.471, $P=0.013$ ). The prognostic value of MTHFD2 for OS was not observed in oligoastrocytoma and oigodendroglioma patients.

\subsection{Functional enrichment analysis}

To further determine the potential function of MTHFD2 in LGG, we performed co-expression analysis using TCGA database, and subsequently got 20161 MTHFD2-associated 
genes. 9347 genes were positively associated with MTHFD2 and the other 10814 genes were negatively correlated with MTHFD2. The MTHFD2-associated genes with absolute correlation $>0.5$ and $P<0.05$ were selected for enrichment analysis.

Table.3 GO annotation analysis

\begin{tabular}{|c|c|c|}
\hline Category & Terms & $P$-value \\
\hline \multicolumn{3}{|l|}{ Positive-related genes } \\
\hline molecular function & Transcription factor activity, sequence-specific DNA binding & $4.74-2$ \\
\hline (MF) & Protein binding & 7.2E-2 \\
\hline \multirow{4}{*}{$\begin{array}{l}\text { biological process } \\
\text { (BP) }\end{array}$} & Positive regulation of endothelial cell differentiation & $2.3 \mathrm{E}-2$ \\
\hline & Positive regulation of apoptosis process & $2.6 \mathrm{E}-2$ \\
\hline & Negative regulation of cell growth & $2.8 \mathrm{E}-2$ \\
\hline & Regulation of small GTPase mediated signal transduction & 3.3E-2 \\
\hline \multirow{3}{*}{$\begin{array}{c}\text { cellular component } \\
\text { (CC) }\end{array}$} & Negative regulation of myeloid cell differentiation & 4.0E-2 \\
\hline & cytosol & $2.1 \mathrm{E}-2$ \\
\hline & nucleus & 4.3E-2 \\
\hline \multicolumn{3}{|l|}{ Negative-related genes } \\
\hline \multirow{4}{*}{$\begin{array}{l}\text { molecular function } \\
\qquad(\mathrm{MF})\end{array}$} & GDP binding & $9.2 \mathrm{E}-3$ \\
\hline & Cellular response to hypoxia & $9.6 \mathrm{E}-3$ \\
\hline & Triglyceride catabolic process & $3.8 \mathrm{E}-2$ \\
\hline & Negative regulation of signal transduction & $5.1 \mathrm{E}-2$ \\
\hline \multirow{3}{*}{$\begin{array}{l}\text { biological process } \\
\text { (BP) }\end{array}$} & Cellular response to hypoxia & $9.6 \mathrm{E}-3$ \\
\hline & Triglyceride catabolic process & $3.8 \mathrm{E}-2$ \\
\hline & Negative regulation of signal transduction & $5.1 \mathrm{E}-2$ \\
\hline cellular component & Apical plasma membrane & $1.3 \mathrm{E}-2$ \\
\hline (CC) & T-tubule & $6.3 \mathrm{E}-2$ \\
\hline
\end{tabular}

As shown in Table.3, GO-MF analysis suggested that positive-related genes are mainly involved in transcription factor activity and sequence-specific DNA binding, and negative-related genes are enriched in GDP binding, cellular response to hypoxia and triglyceride catabolic process For BP, positive-related genes participated in positive regulation of endothelial cell differentiation, positive regulation of apoptosis process, negative regulation of cell growth and regulation of small GTPase mediated signal transduction. Negative-related genes were associated with cellular response to hypoxia and triglyceride catabolic process. In addition, GO-CC analysis displayed that positive-related genes were significantly enriched in cytosol and nucleus, and negative-related genes were mainly enriched in apical plasma membrane. 


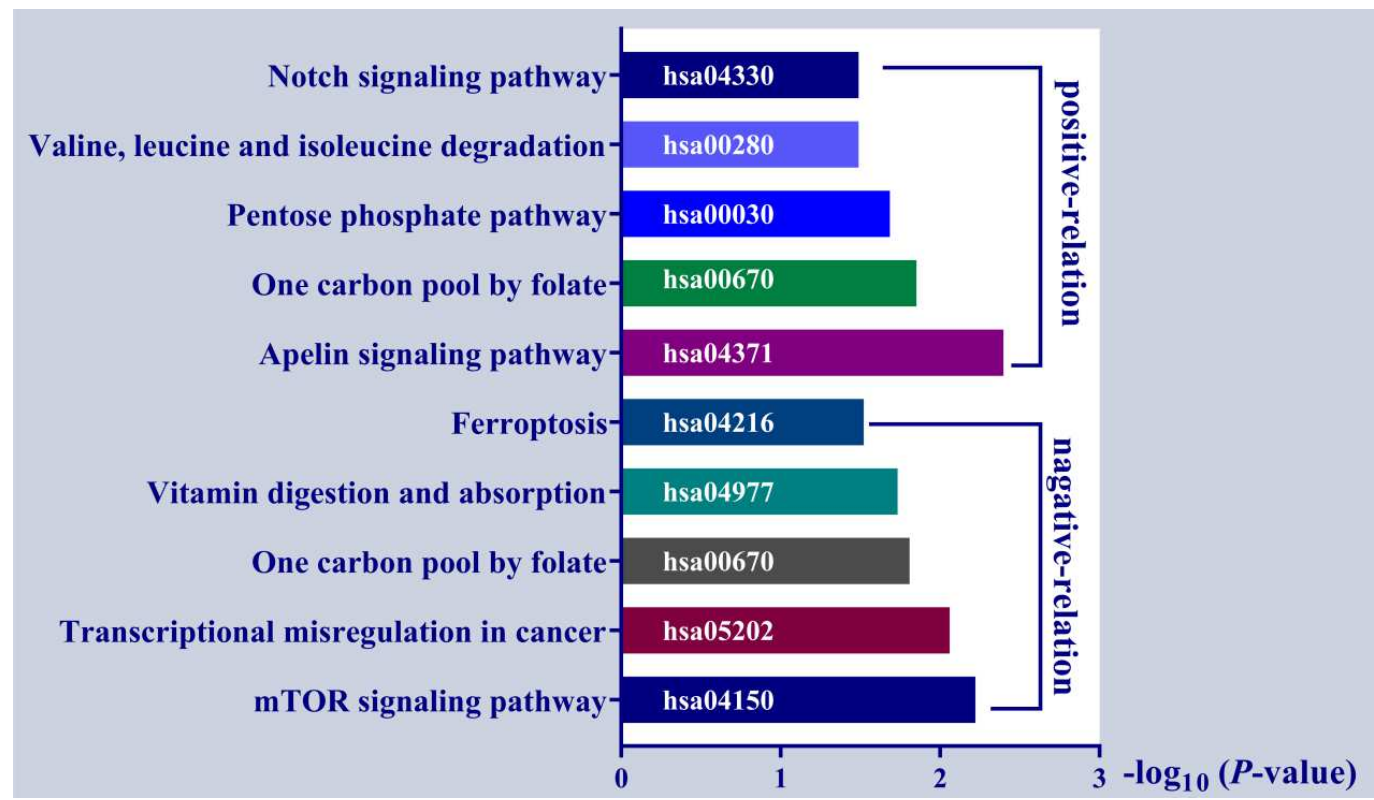

Fig.7 KEGG pathway analysis on co-expressed genes. Calculated genes showing positive and negative correlation with MTHFD2 were analyzed, respectively, and the top 5 significant pathways were presented.

KEGG pathway analysis (Fig.7) indicated that positive-related genes significantly participates in apelin signaling pathway, one carbon pool by folate, pentose phosphate pathway, valine, leucine and isoleucine degradation, and notch signaling pathway. While the negative-related genes correlated with mTOR signaling pathway, transcriptional misregulation in cancer, one carbon pool by folate, vitamin digestion and absorption, and ferroptosis.

\section{Discussion}

Previous study has suggested that MTHFD2 might play an important role and potentially be valuable in the prognosis and treatment of glioma [16]. However, detailed function of MTHFD2 in LGG has not been well-examined. The present study initially explored the potential role of MTHFD2 in LGG through bioinformatics analysis. We found that MTHFD2 was highly expressed in most of human cancers including LGG. The expression of MTHFD2 was associated with sub-types, TP53 mutation status and age of LGG patients. Age, radiation therapy status, histologic grade and sub-types were able to influence the prognosis of LGG patients.

Several researches have showed that MTHFD2 is frequently highly expressed in various tumors and may serve as a tumor promoter by promoting cells proliferation. MTHFD2 was found highly expressed in colorectal cancer (CRC) cells, and silence of MTHFD2 expression inhibited the proliferation, weakened the migration ability and promoted the apoptosis of CRC cells [17]. MTHFD2 was also significantly over-expressed in non-small cell lung cancer (NSCLC) tissues, and knockdown of MTHFD2 resulted in reduction of tumor cell growth and tumorigenicity [18]. In addition, MTHFD2 over-expression was associated with cell proliferation and vimentin-modulated migration and invasion in renal cell carcinoma (RCC) [19]. MTHFD2 expression was significantly associated with the clinical outcome of patients, and high expression of MTHFD2 resulted in poor prognosis in hepatocellular carcinoma [20], esophageal squamous cell carcinoma [21] and breast cancer [22]. Contrary to previous studies, however, our research desirably found that MTHFD2 high expression is favorable to the clinical outcome of LGG patients and achieves a good prognosis. As far as we are aware, no research reported the positive 
prognostic effects of MTHFD2 high expression on human cancers up to now.

For better understanding the function of MTHFD2 in LGG, we performed pathway enrichment analysis to reveal the MTHFD2-related pathway involved in LGG. The results indicated that the positive-related genes with MTHFD2 are mainly enriched in apelin signaling pathway and one-carbon pool by folate in LGG. Apelin was found to attenuate early brain injury via suppressing neuronal apoptosis through the PI3K/Akt signaling [23]. Apelin mediated neuroprotective effects by regulating oxidative stress, autophagy and apoptosis [24], as well as alleviated neuroinflammation [25]. It was possible that the neuroprotective effects of MTHFD2-associated apelin signaling pathway may play a vital role in LGG development. Folate-mediated one-carbon metabolism plays a central role in a broad array of metabolic processes required for the survival and growth of tumor cells [26]. Folate was found to stimulate the proliferation of acute lymphocytic leukemia (ALL) cell, but the ALL patients with aminopterin treatment, an intermediate of folate metabolism, showed clinical, hematologic and pathological evidence of improvement [27]. Therefore, it was inferred that MTHFD2 is involved in the folate metabolism, influences its intermediate production and thereby regulates the progression of LGG.

Moreover, the negative-related genes with MTHFD2 were associated with mTOR signaling pathway and transcriptional misregulation in cancer. mTOR is strongly activated in microglia, and mTOR-deficient microglia lost their typical proliferative and inflammatory responses to excitatory injury, as well as increase the neuronal loss. Microglial mTOR played a protective role in mitigating neuronal loss and was strongly implicated in attenuating epileptogenesis [28]. Activation of mTOR signaling pathway can reduce brain damage, inhibit neuronal apoptosis and autophagy [29]. MTHFD2-associated mTOR signaling pathway might exert protective effects on neuronal development in LGG. There was related study which suggests the novel function of MTHFD2 in RNA metabolism and translation, identifying as a potential non-enzymatic functions [30]. It follows that MTHFD2 has the potential to play a significant role in LGG through various pathways.

We further found that MTHFD2 high expression increases the survival time of LGG patients for both male and female. In addition, high expression of MTHFD2 facilitated clinical outcome of those patients with age $\geq 45$ years old, receiving radiation therapy and grade 3 . The prognostic effects of MTHFD2 expression differed in different sub-types of LGG. Cox regression indicated that MTHFD2 expression remains as an independent prognostic factor for OS of LGG patients, especially for patients with astrocytoma. It seemed that MTHFD2 expression exerts a positive role in LGG patients and may act as a potential therapeutic target for LGG treatment.

Recent studies have highlighted the critical value of MTHFD2 as a therapeutic target for treatment of cancers. Xu et, al suggested that microRNA-940 could inhibit glioma progression by blocking mitochondrial folate metabolism through targeting of MTHFD2 [31]. Yam et, al indicated that microRNA-33a-5p suppresses the growth and migration of colorectal cancer cell by inhibiting MTHFD2 [32]. Li et, al reported that upregulation of miR-504-3p is associated with favorable prognosis of acute myeloid leukemia and may serve as a tumor suppressor by targeting MTHFD2 [33]. Thus it can be seen that MTHFD2 is expected to become an effective target for more cancer treatment including LGG. 


\section{Conclusions}

The current study, based on a bioinformatics analysis from TCGA database, demonstrated that the expression of MTHFD2 is significantly increased in LGG, and associated with age, sub-types and TP53-mutation status of patients. High expression of MTHFD2 favorably increased the patient's survival time, and MTHFD2 was determined as a novel prognostic biomarker for OS of LGG patients. In addition, MTHFD2 was strongly implicated in LGG through mediating various pathways such as mTOR signaling pathway and apelin pathway. Our research emphasized the importance of MTHFD2 in LGG, and the experimental intervention must be icing on the cake for this research.

Abbreviations: MTHFD2: Methylenetetrahydrofolate dehydrogenase 2; LGG: low-grade gliomas: GO: gene ontology; KEGG: Kyoto Encyclopedia of Genes and Genomes; OS: overall survival; BP: biological processes; MF: molecular Function; CC: cellular component;

Acknowledgement: Not applicable.

Authors' contributions: LFS: research design and manuscript writing. QZ: figures and/or tables preparation. XYS: data collection and analysis. HJN: drafts of the paper review. All authors read and approved the final manuscript.

Funding: This work was supported by the Zhejiang Provincial Department of Health Project (No. 201466094).

Availability of data and materials: The datasets used and/or analyzed during the current study are available from the corresponding authors upon reasonable request.

Ethics approval and consent to participate: Not applicable.

Consent for publication: Not applicable.

Competing interests: The authors report no competing interests in this work.

\section{References}

1. Ostrom QT, Gittleman H, Liao P, Rouse C, Chen Y, Dowling J, Wolinsky Y, Kruchko C, Barnholtz-Sloan J: CBTRUS statistical report: primary brain and central nervous system tumors diagnosed in the United States in 2007-2011. Neuro Oncol 2014, 16 Suppl 4:iv1-63.

2. Chen W, Zheng R, Baade PD, Zhang S, Zeng H, Bray F, Jemal A, Yu XQ, He J: Cancer statistics in China, 2015. CA Cancer J Clin 2016, 66:115-132.

3. Louis DN, Perry A, Reifenberger G, von Deimling A, Figarella-Branger D, Cavenee WK, Ohgaki H, Wiestler OD, Kleihues P, Ellison DW: The 2016 World Health Organization Classification of Tumors of the Central Nervous System: a summary. Acta Neuropathol 2016, 131:803-820.

4. Louis DN, Ohgaki H, Wiestler OD, Cavenee WK, Burger PC, Jouvet A, Scheithauer BW, Kleihues P: The 2007 WHO classification of tumours of the central nervous system. Acta Neuropathol 2007, 114:97-109.

5. Gnekow AK, Walker DA, Kandels D, Picton S, Giorgio P, Grill J, Stokland T, Sandstrom PE, Warmuth-Metz M, Pietsch T, et al: A European randomised controlled trial of the addition of etoposide to standard vincristine and carboplatin induction as part of an 18-month 
treatment programme for childhood ( $</=\mathbf{1 6}$ years) low grade glioma - A final report. Eur J Cancer 2017, 81:206-225.

6. Forst DA, Nahed BV, Loeffler JS, Batchelor TT: Low-grade gliomas. Oncologist 2014, 19:403-413.

7. Morshed RA, Young JS, Hervey-Jumper SL, Berger MS: The management of low-grade gliomas in adults. $J$ Neurosurg Sci 2019, 63:450-457.

8. Afra D, Osztie E, Sipos L, Vitanovics D: Preoperative history and postoperative survival of supratentorial low-grade astrocytomas. Br J Neurosurg 1999, 13:299-305.

9. Tibbetts AS, Appling DR: Compartmentalization of Mammalian folate-mediated one-carbon metabolism. Anпи Rev Nutr 2010, 30:57-81.

10. Gustafsson R, Jemth AS, Gustafsson NM, Farnegardh K, Loseva O, Wiita E, Bonagas N, Dahllund L, Llona-Minguez S, Haggblad M, et al: Crystal Structure of the Emerging Cancer Target MTHFD2 in Complex with a Substrate-Based Inhibitor. Cancer Res 2017, 77:937-948.

11. Tedeschi PM, Vazquez A, Kerrigan JE, Bertino JR: Mitochondrial Methylenetetrahydrofolate Dehydrogenase (MTHFD2) Overexpression Is Associated with Tumor Cell Proliferation and Is a Novel Target for Drug Development. Mol Cancer Res 2015, 13:1361-1366.

12. Nishimura T, Nakata A, Chen X, Nishi K, Meguro-Horike M, Sasaki S, Kita K, Horike SI, Saitoh K, Kato K, et al: Cancer stem-like properties and gefitinib resistance are dependent on purine synthetic metabolism mediated by the mitochondrial enzyme MTHFD2. Oncogene 2019, 38:2464-2481.

13. Koufaris C, Valbuena GN, Pomyen Y, Tredwell GD, Nevedomskaya E, Lau CH, Yang T, Benito A, Ellis JK, Keun HC: Systematic integration of molecular profiles identifies miR-22 as a regulator of lipid and folate metabolism in breast cancer cells. Oncogene 2016, 35:2766-2776.

14. Pikman Y, Puissant A, Alexe G, Furman A, Chen LM, Frumm SM, Ross L, Fenouille N, Bassil CF, Lewis CA, et al: Targeting MTHFD2 in acute myeloid leukemia. $J$ Exp Med 2016, 213:1285-1306.

15. Lehtinen L, Ketola K, Makela R, Mpindi JP, Viitala M, Kallioniemi O, Iljin K: High-throughput RNAi screening for novel modulators of vimentin expression identifies MTHFD2 as a regulator of breast cancer cell migration and invasion. Oncotarget 2013, 4:48-63.

16. Liu M, Xu Z, Du Z, Wu B, Jin T, Xu K, Xu L, Li E, Xu H: The Identification of Key Genes and Pathways in Glioma by Bioinformatics Analysis. J Immunol Res 2017, 2017:1278081.

17. Wei Y, Liu P, Li Q, Du J, Chen Y, Wang Y, Shi H, Wang Y, Zhang H, Xue W, et al: The effect of MTHFD2 on the proliferation and migration of colorectal cancer cell lines. Onco Targets Ther 2019, 12:6361-6370.

18. Yu C, Yang L, Cai M, Zhou F, Xiao S, Li Y, Wan T, Cheng D, Wang L, Zhao C, Huang X: Down-regulation of MTHFD2 inhibits NSCLC progression by suppressing cycle-related genes. J Cell Mol Med 2020, 24:1568-1577.

19. Lin H, Huang B, Wang H, Liu X, Hong Y, Qiu S, Zheng J: MTHFD2 Overexpression Predicts Poor Prognosis in Renal Cell Carcinoma and is Associated with Cell Proliferation and Vimentin-Modulated Migration and Invasion. Cell Physiol Biochem 
2018, 51:991-1000.

20. Liu X, Huang Y, Jiang C, Ou H, Guo B, Liao H, Li X, Yang D: Methylenetetrahydrofolate dehydrogenase 2 overexpression is associated with tumor aggressiveness and poor prognosis in hepatocellular carcinoma. Dig Liver Dis 2016, 48:953-960.

21. $\mathrm{He} \mathrm{H,} \mathrm{Li} \mathrm{PC,} \mathrm{Jia} \mathrm{W,} \mathrm{Hu} \mathrm{B,} \mathrm{Ji} \mathrm{CS:} \mathrm{High} \mathrm{Expression} \mathrm{of} \mathrm{Methylenetetrahydrofolate}$ Dehydrogenase 2 (MTHFD2) in Esophageal Squamous Cell Carcinoma and its Clinical Prognostic Significance. Med Sci Monit 2020, 26:e920259.

22. Liu F, Liu Y, He C, Tao L, He X, Song H, Zhang G: Increased MTHFD2 expression is associated with poor prognosis in breast cancer. Tumour Biol 2014, 35:8685-8690.

23. Liu Y, Zhang T, Wang Y, Wu P, Li Y, Wang C, Xu S, Shi H: Apelin-13 attenuates early brain injury following subarachnoid hemorrhage via suppressing neuronal apoptosis through the GLP-1R/PI3K/Akt signaling. Biochem Biophys Res Commun 2019, 513:105-111.

24. Zhu J, Gao W, Shan X, Wang C, Wang H, Shao Z, Dou S, Jiang Y, Wang C, Cheng B: Apelin-36 mediates neuroprotective effects by regulating oxidative stress, autophagy and apoptosis in MPTP-induced Parkinson's disease model mice. Brain Res 2020, 1726: 146493 .

25. Zhang ZX, Li E, Yan JP, Fu W, Shen P, Tian SW, You Y: Apelin attenuates depressive-like behavior and neuroinflammation in rats co-treated with chronic stress and lipopolysaccharide. Neuropeptides 2019, 77:101959.

26. Green NH, Galvan DL, Badal SS, Chang BH, LeBleu VS, Long J, Jonasch E, Danesh FR: MTHFD2 links RNA methylation to metabolic reprogramming in renal cell carcinoma. Oncogene 2019, 38:6211-6225.

27. Farber S, Diamond LK: Temporary remissions in acute leukemia in children produced by folic acid antagonist, 4-aminopteroyl-glutamic acid. $N$ Engl J Med 1948, 238:787-793.

28. Zhao XF, Liao Y, Alam MM, Mathur R, Feustel P, Mazurkiewicz JE, Adamo MA, Zhu XC, Huang Y: Microglial $m$ TOR is neuronal protective and anti-epileptogenic in the pilocarpine model of temporal lobe epilepsy. J Neurosci 2020.

29. Zhang Y, He Q, Yang M, Hua S, Ma Q, Guo L, Wu X, Zhang C, Fu X, Liu J: Dichloromethane extraction from Piper nigrum $L$. and P. longum $L$. to mitigate ischemic stroke by activating the AKT/mTOR signaling pathway to suppress autophagy. Brain Res 2020:147047.

30. Koufaris C, Nilsson R: Protein interaction and functional data indicate MTHFD2 involvement in RNA processing and translation. Cancer Metab 2018, 6:12.

31. Xu T, Zhang K, Shi J, Huang B, Wang X, Qian K, Ma T, Qian T, Song Z, Li L: MicroRNA-940 inhibits glioma progression by blocking mitochondrial folate metabolism through targeting of MTHFD2. Am J Cancer Res 2019, 9:250-269.

32. Yan Y, Zhang D, Lei T, Zhao C, Han J, Cui J, Wang Y: MicroRNA-33a-5p suppresses colorectal cancer cell growth by inhibiting MTHFD2. Clin Exp Pharmacol Physiol 2019, 46:928-936.

33. Li SM, Zhao YQ, Hao YL, Liang YY: Upregulation of miR-504-3p is associated with favorable prognosis of acute myeloid leukemia and may serve as a tumor suppressor by targeting MTHFD2. Eur Rev Med Pharmacol Sci 2019, 23:1203-1213. 
Figures

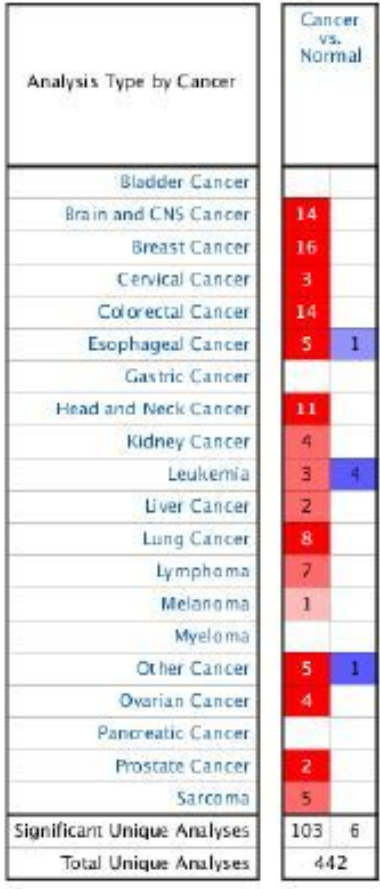

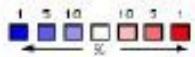
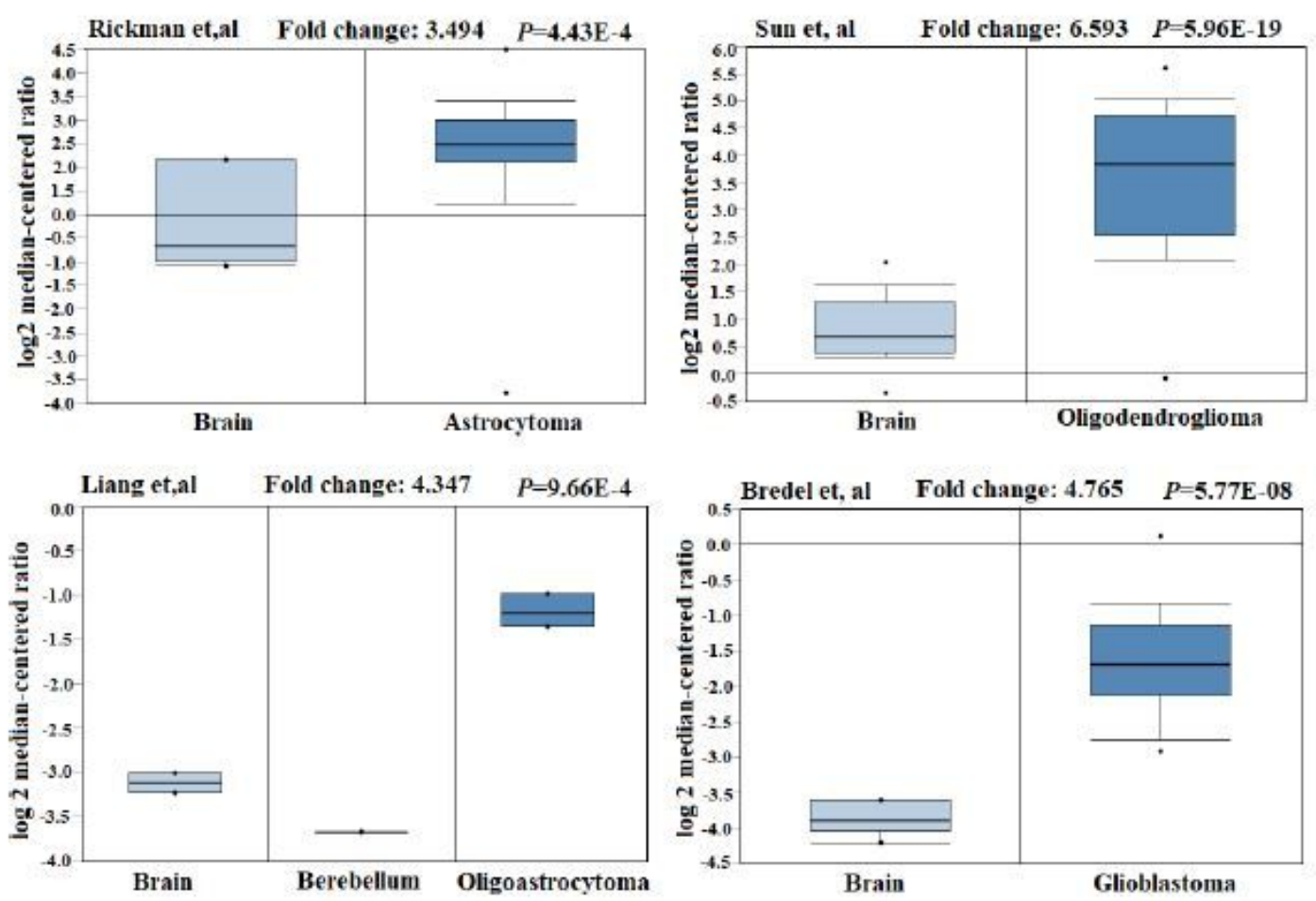

(B)

(A)

\section{Figure 1}

Expression analysis on MTHFD2. (A) disease summary for MTHFD2; (B) MTHFD2 expression in normal and brain cancers. The threshold was set by P-value: 0.05 , fold change: 2 , gene rank: TOP $10 \%$. The data was obtained from Oncomine database. 


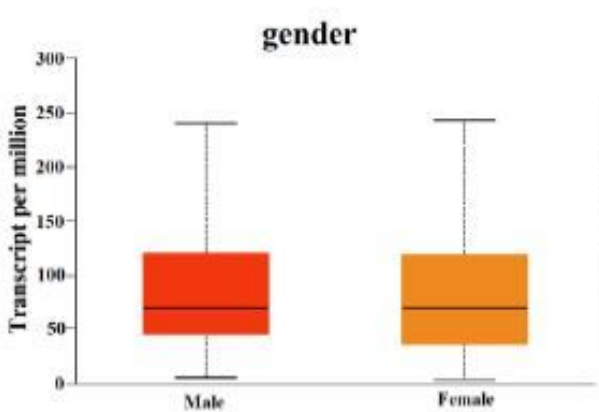

(A)

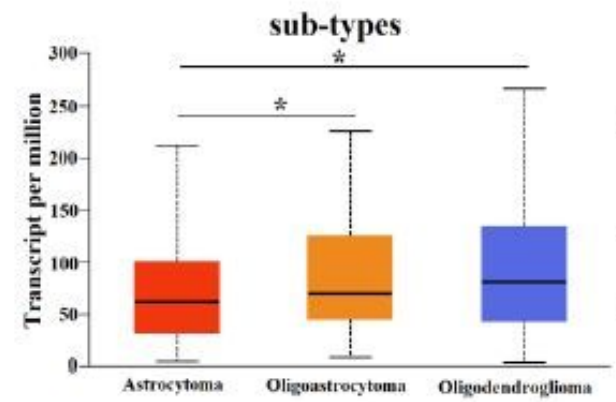

(D)

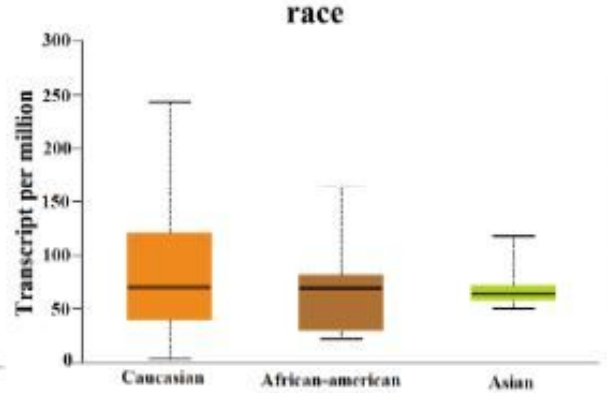

(B)

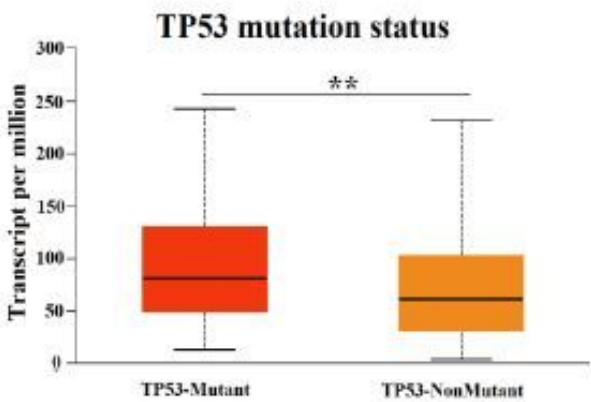

(E)

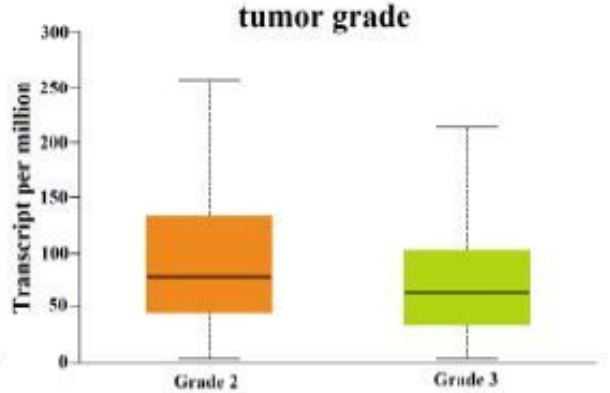

(C)

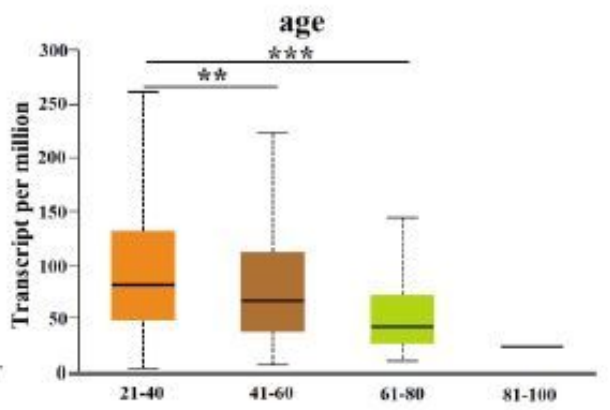

(F)

\section{Figure 2}

MTHFD2 expression profile based on clinical phenotype in LGG. (A) gender; (B) race; (C) tumor grade; (D) subsub-types; (E) TP53 mutation status; $(F)$ age. types; ${ }^{*} P<0.05$, ${ }^{*} P<0.01$ and ${ }^{*} * * P<0.001$
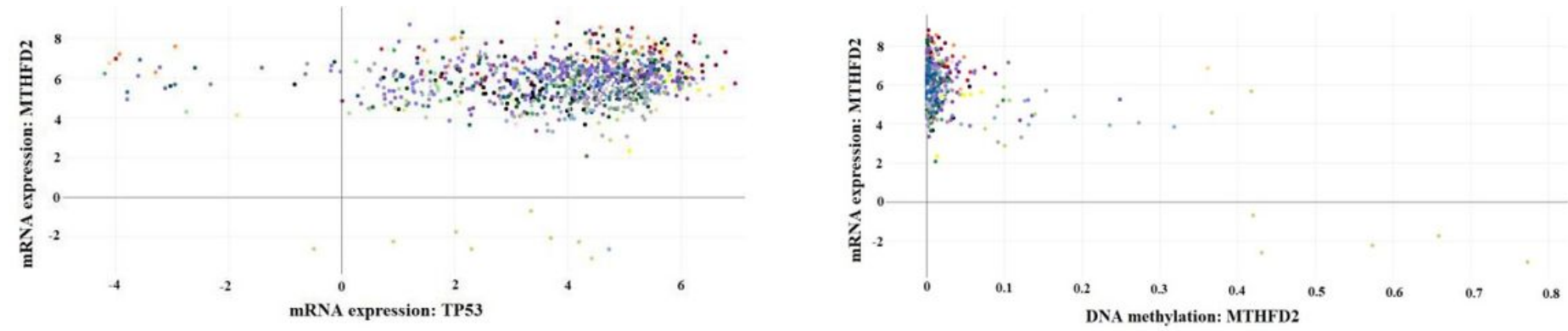

\section{Figure 3}

The expression of MTHFD2 with (A) TP53 expression and B) MTHFD2 methylation. 


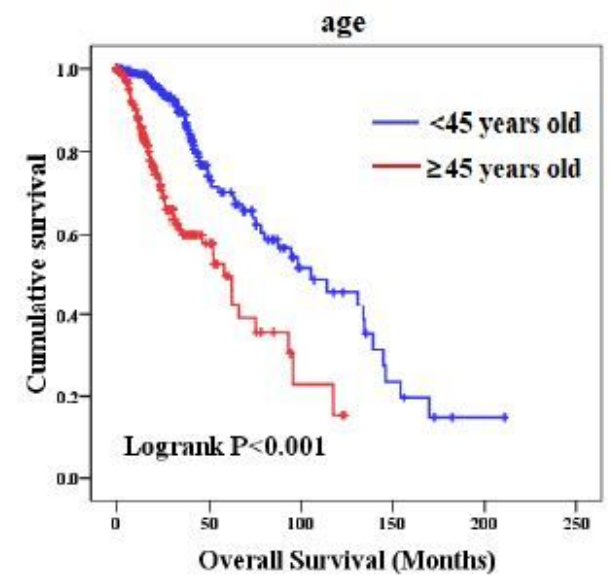

(A)

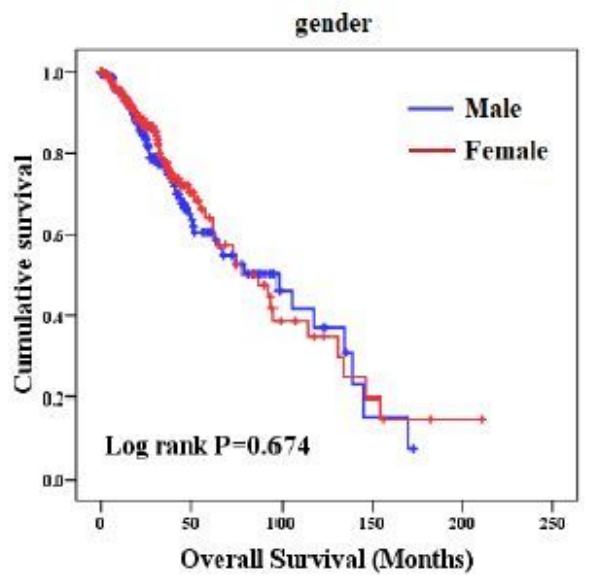

(B)

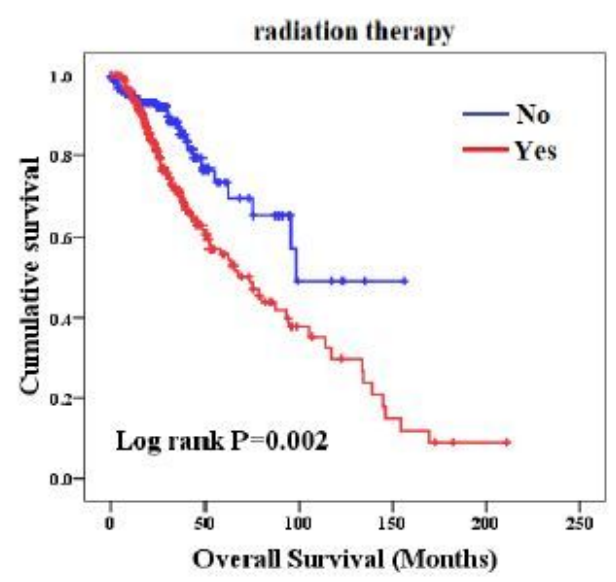

(C)

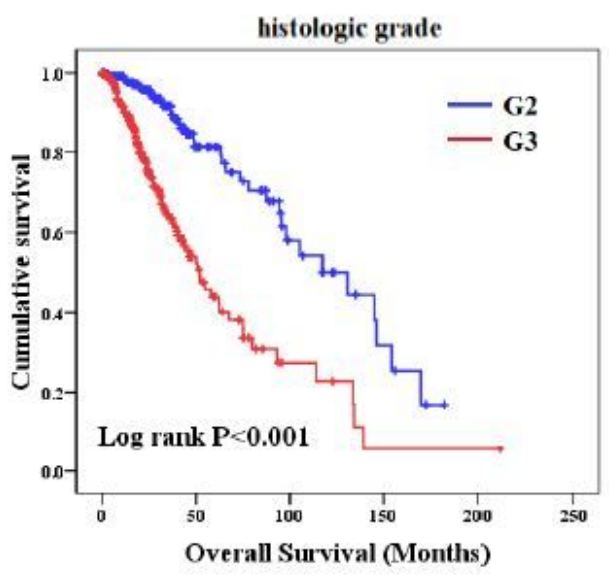

(D)

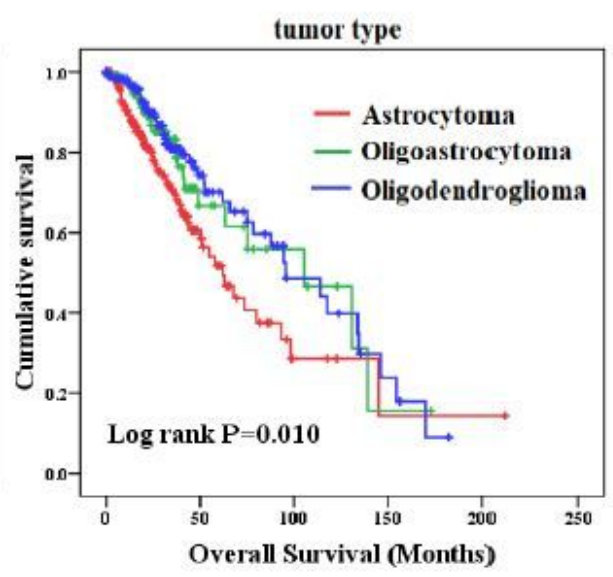

(E)

\section{Figure 4}

The association of clinical phenotype with OS in LGG patient. (A) age; (B) gender; (C) radiation therapy; (D) histologic grade; (E) tumor type. 


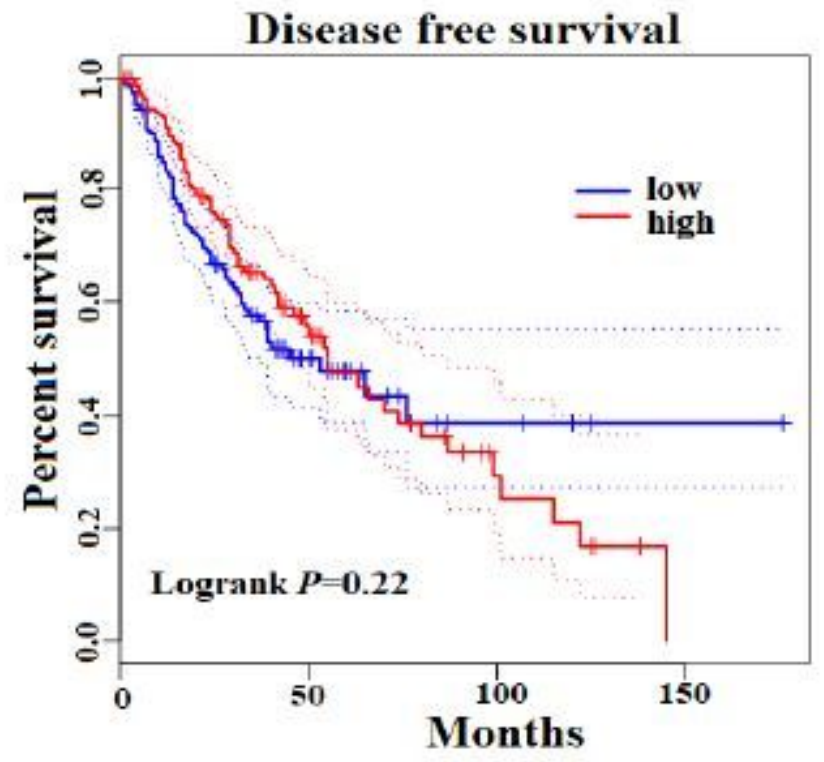

(A)

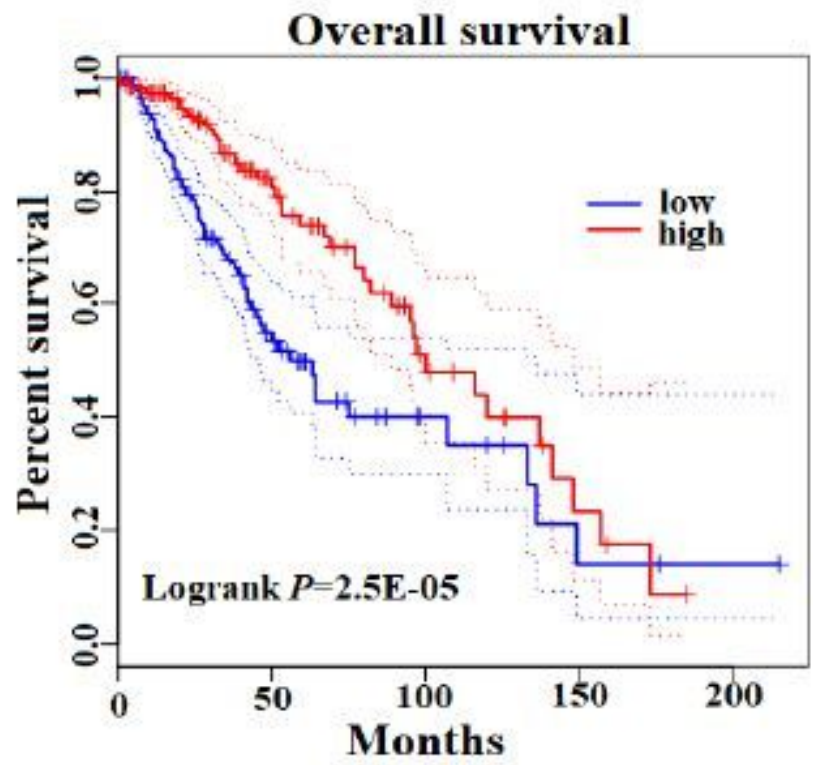

(B)

Figure 5

The association of MTHFD2 expression with prognosis LGG patients. (A) disease free survival (DFS): (B) Overall survival (OS). 

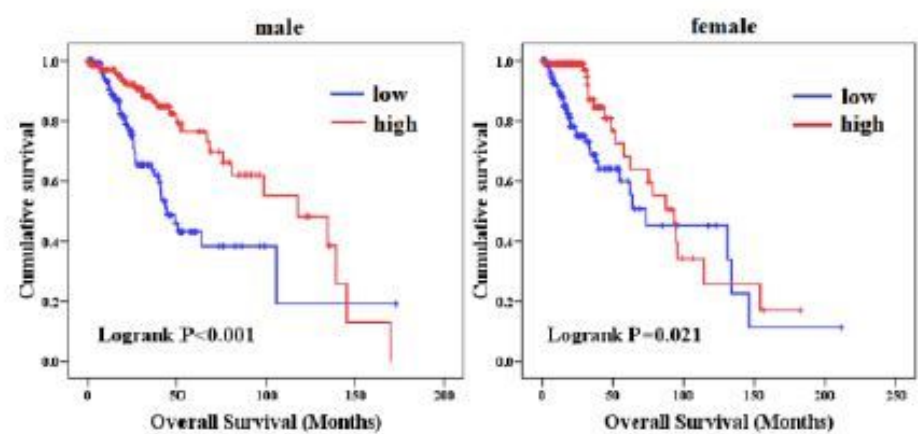

(A)
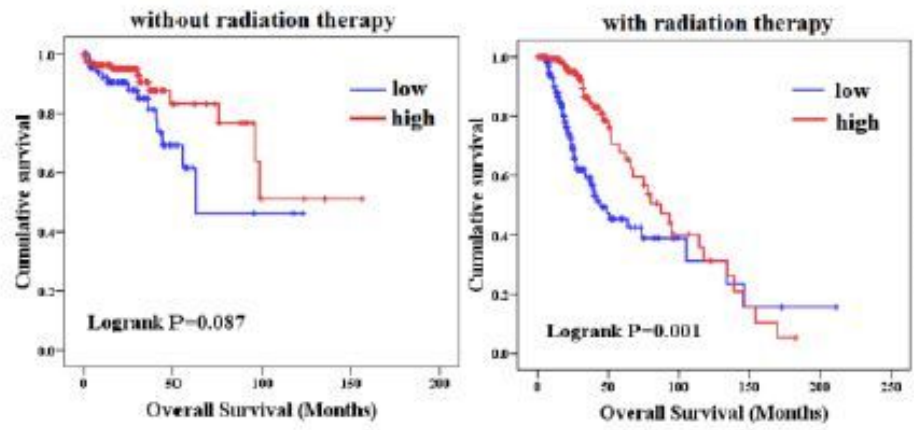

(C)
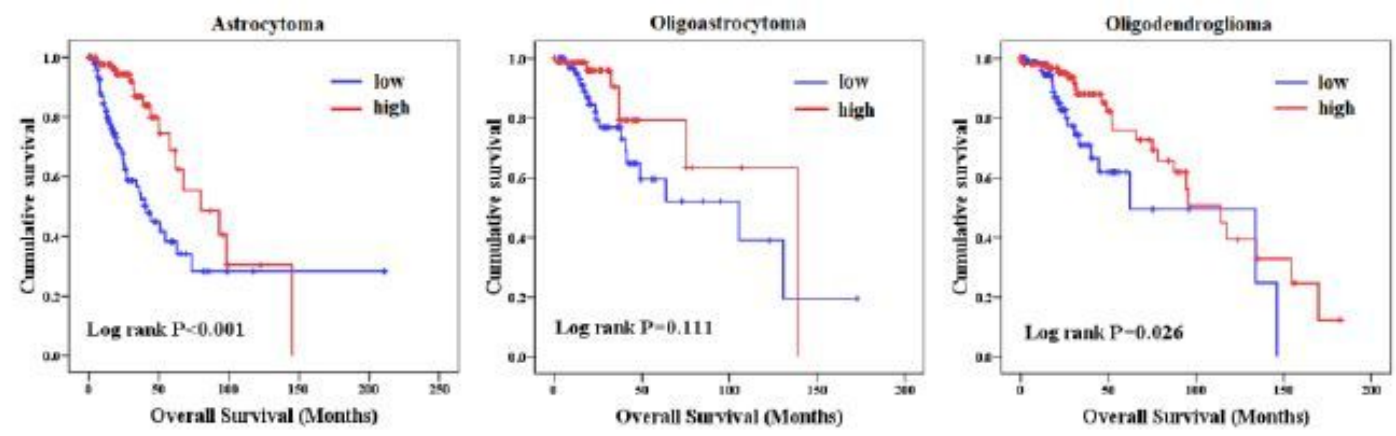

(E) age $<45$ years old

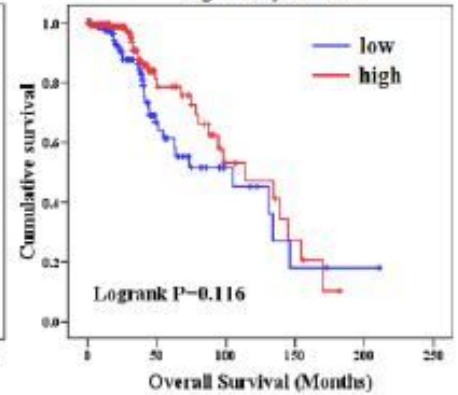

(B)
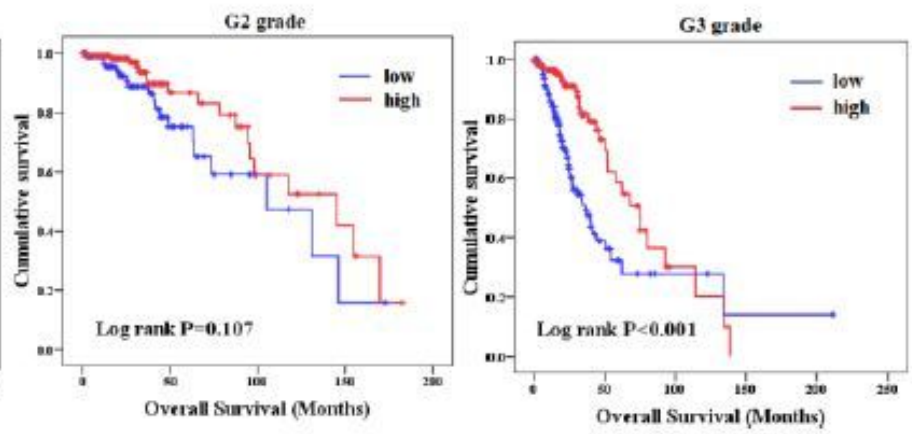

(D)

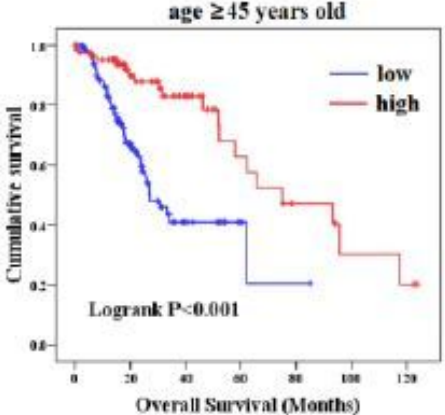




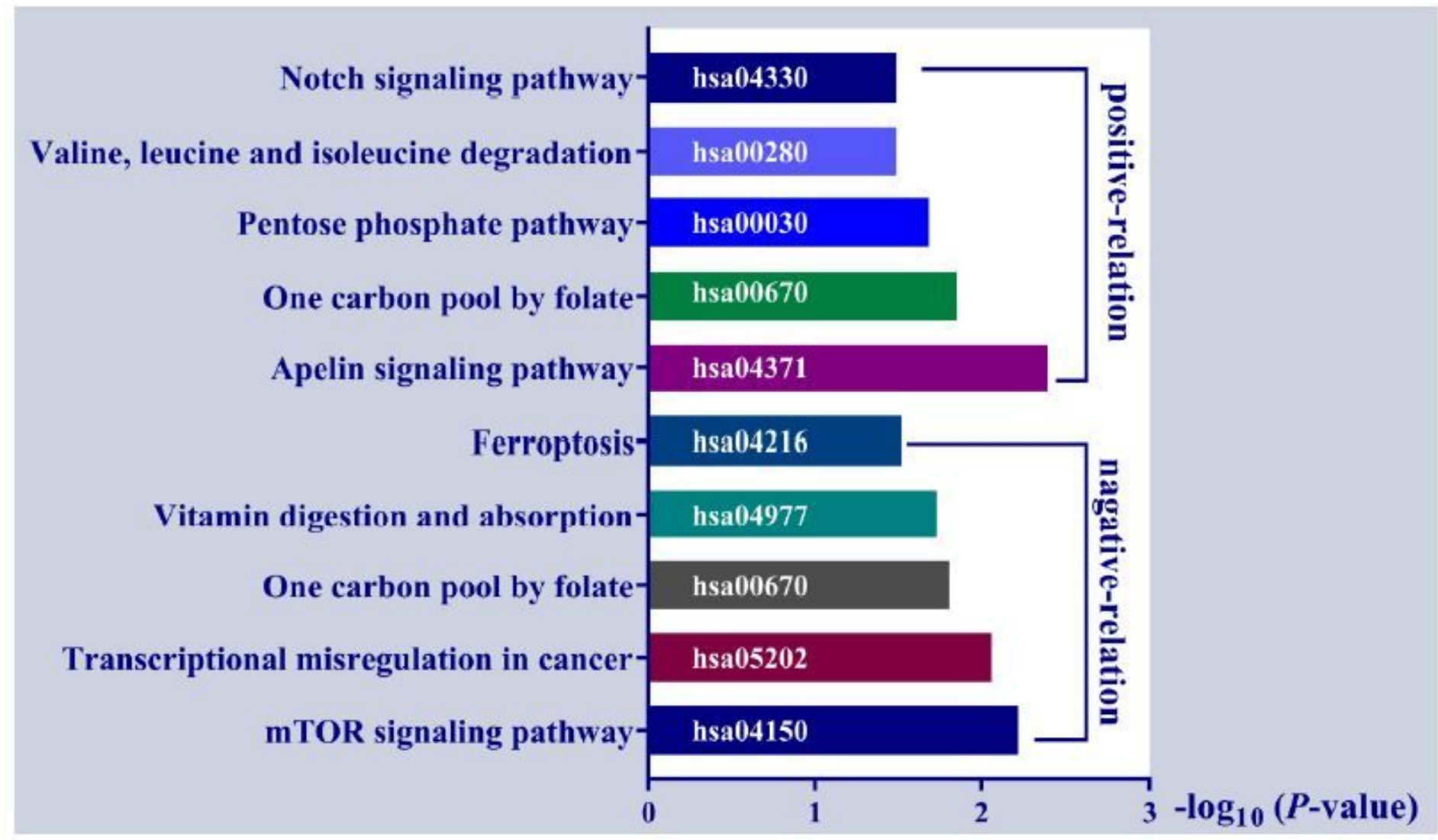

Figure 7

KEGG pathway analysis on co-expressed genes. Calculated genes showing positive and negative correlation with MTHFD2 were analyzed, respectively, and the top 5 significant pathways presented. 